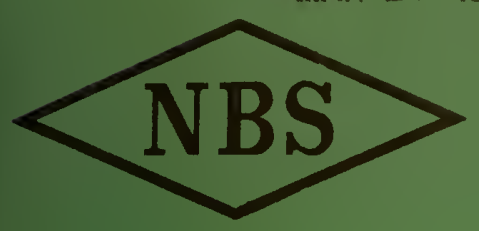
taken from the library.

Eechnical Mote

\title{
MICROWAVE REFLECTION TECHNIQUES FOR DENSE PLASMA DIAGNOSTICS
}

S. TAKEDA AND T. TSUKISHIMA

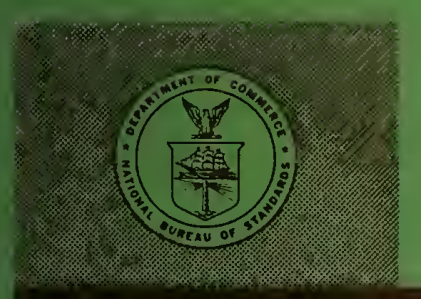

U. S. DEPARTMENT OF COMMERCE NATIONAL BUREAU OF STANDARDS 


\section{THE NATIONAL BUREAU OF STANDARDS}

The National Bureau of Standards is a principal focal point in the Federal Government for assuring maximum application of the physical and engineering sciences to the advancement of technology in industry and commerce. Its responsibilities include development and maintenance of the national standards of measurement, and the provisions of means for making measurements consistent with those standards; determination of physical constants and properties of materials; development of methods for testing materials, mechanisms, and structures, and making such tests as may be necessary, particularly for government agencies; cooperation in the establishment of standard practices for incorporation in codes and specifications; advisory service to governinent agencies on scientific and technical problems; invention and development of devices to serve special needs of the Government; assistance to industry, business, and consumers in the development and acceptance of commercial standards and simplified trade practice recommendations; administration of programs in cooperation with United States business groups and standards organizations for the development of international standards of practice; and maintenance of a clearinghouse for the collection and dissemination of scientific, technical, and engineering information. The scope of the Bureau's activities is suggested in the following listing of its four Institutes and their organizational units.

Institute for Basic Standards. Electricity. Metrology. Heat. Radiation Physics. Mechanics. Applied Mathematics. Atomic Physics. Physical Chemistry. Laboratory Astrophysics.* Radio Standards Laboratory: Radio Standards Physics; Radio Standards Engineering. ${ }^{*}$ Office of Standard Reference Data.

Institute for Materials Research. Analytical Chemistry. Polymers. Metallurgy. Inorganic Materials. Reactor Radiations. Cryogenics. * Office of Standard Reference Materials.

Central Radio Propagation Laboratory.* Ionosphere Research and Propagation. Troposphere and Space Telecommunications. Radio Systems. Upper Atmosphere and Space Physics.

Institute for Applied Technology. Textiles and Apparel Technology Center. Building Research. Industrial Equipment. Information Technology. Performance Test Development. Instrumentation. Transport Systems. Office of Technical Services. Office of Weights and Measures. Office of Engineering Standards. Office of Industrial Services.

\footnotetext{
* NBS Group, Joint Institute for Laboratory Astrophysics at the University of Colorado.

** Located at Boulder, Colorado.
} 


\title{
NATIONAL BUREAU OF STANDARDS Technical Note 256
}

ISSUED MARCH 10, 1965

\section{MICROWAVE REFLECTION TECHNIQUES \\ FOR DENSE PLASMA DIAGNOSTICS}

\author{
S. Takeda and T. Tsukishima
}

NBS Technical Notes are designed to supplement the Bureau's regular publications program. They provide a means for making available scientific data that are of transient or limited interest. Technical Notes may be listed or referred to in the open literature. 


\section{Contents}

page

1. Introduction ... . . . . . . . . . . . . . . . 1

2. Principle of Reflection Method . . . . . . . . . . . . 3

3. Some Considerations for Reducing Experimental Errors . . . 8 3.1 Difference Method . . . . . . . . . . . . . 8

3.2 Effect of Power Losses Along the Path Between the Plasma Boundary and the Standing Wave Detectors . . . 10

4. Further Extension of Measurable Density Range . . . . • . 11

5. Microwave Reflection Probe . . . . . . . . . . . . . 14

6. Reflection by Inhomogeneous Plasma . . . . . . . . . . . 17

7. Reflection by Boundary Layer with Density Gradient . . . . 20

8. Concluding Remarks . . . . . . . . . . . . . . . 22

9. Acknowledgment . . . . . . . . . . . . . . . 23

10. References................... . . . 24 


\section{Microwave Reflection Techniques for Dense Plasma Diagnostics}

Susumu Takeda* and Takashige Tsukishima

The microwave reflection method for measuring the electron density whose plasma frequency is higher than the probing frequency is described in detail. Various expressions and formulas which are useful for a variety of experimental conditions are given. A method to extend further the measurable density range is proposed. The fringing field effects are considered when a waveguide is used as a probe. References are given to the experimental works which substantiate the theoretical analyses. Also included are the analyses of reflection by a non-uniform boundary and also by an inhomogeneous plasma.

\section{Introduction}

The determination of the electron density, including its spatial distribution, has been one of the first requisites in plasma researches. Among other methods, microwave techniques have been extensively used because of great simplicity in handling and interpreting the results. The microwave methods hitherto developed may be divided into 1) the cavity method and 2) the propagation method which is applicable to plasmas in a waveguide as well as in free space. The cavity method and the waveguide propagation method are mainly used for fundamental studies of small scale laboratory plasmas, while the free space propagation method has been widely used in various high-temperature devices. A shortcoming of the conventional transmission method is the basic difficulty of investigating the density distribution, as well as a certain cumbersomeness of the arrangements. Another difficulty resides

*Present address, Nagoya University, Nagoya, Japan 
in the fact that an electron density whose plasma frequency exceeds the probing frequency is not measurable. Thus, even with $3 \mathrm{~mm}$ waves the maximum measurable density is $10^{14} / \mathrm{cc}$. In order to overcome these difficulties, a new reflection method was proposed by one of the authors. ${ }^{1}$ While the conventional reflection method or cutoff method is only for locating plasma, ${ }^{2}$ the present method admits measuring an electron density higher than critical. The method is thus different also from that of S. C. Lin et al., ${ }^{3}$ who determined sub-critical densities by measuring reflected power. The principle is based on the fact that the electron density of a uniform plasma bounded by a plane boundary is related, in an extremely simple manner, to the phase angle of the reflected wave at the boundary. Since the interaction between the incident microwave and plasma is limited within a narrow region near the boundary, in other words, the penetration distance is very small when the density is high, the measured value by the present method is essentially local.

The proposed method was first applied successfully to a uniform afterglow plasma produced in an X-band waveguide. ${ }^{4}$ Later, an electron density as high as $10^{15} / \mathrm{cc}$ of a shock produced plasma was also measured with the same X-band ( $9000 \mathrm{Mc} /$ sec. $)$ microwave. ${ }^{5}$ A K-band $\left(35 \mathrm{Gc} / \mathrm{sec}_{\text {. }}\right.$ ) microwave reflection probe has been used to obtain the density profile of CANDLE plasma (A magnetically confined argon arc) at the National Bureau of Standards, Washington, D. C. The values obtained by this microwave probe have fitted remarkably with those obtained by other techniques such as the Stark broadening of $H_{\beta}$ and Langmuir probe. ${ }^{6}$ In the course of these experiments, a number of formulas and 
expression have been derived to account for a variety of boundary conditions. It is the purpose of the present paper to collect these results in one place, although some of them have been published elsewhere. Also included are recent analyses of reflection by a non-uniform boundary and also by an inhomogeneous plasma.

The idea described in Chapter 4 has been proposed by S. Takeda, and the calculations in Chapters 6 and 7 have been made by T. Tsukishima.

\section{Principle of Reflection Method}

A plane electromagnetic wave, $e^{j\left(\omega t-k_{0} \cdot z\right)}$, propagating along the $z$-direction in vacuum with angular frequency $\omega$ and propagation constant $k_{0}$, is reflected by a plane boundary, $z=0$, separating a homogeneous plasma $(z>0)$ and vacuum. The reflection coefficient, $R$, is given by

$$
R=\frac{k_{0}-k}{k_{0}+k},
$$

where

$$
K=\alpha-j \beta
$$

$\alpha$ and $\beta$ are the phase and attenuation constants in the plasma. They are related to plasma parameters,

$$
\begin{aligned}
& \omega_{p}^{2} / \omega^{2} \equiv \eta, \\
& \nu / \omega \equiv \delta,
\end{aligned}
$$

where $\omega_{p}$ and $w$ are the plasma frequency and the frequency of the incident wave, $\nu$ the collision frequency of electrons in the plasma, by 


$$
\begin{aligned}
& \left.\alpha=\frac{k_{0}}{\sqrt{2}}\left[\left\{1-\frac{\eta}{1+\delta^{2}}\right)^{2}+\left(\frac{n \delta}{1+\delta^{2}}\right)^{2}\right\}^{\frac{1}{2}}+\left(1-\frac{\eta}{1+\delta^{2}}\right)\right]^{\frac{1}{2}}, \\
& \beta=\frac{k_{0}}{\sqrt{2}}\left[\left\{\left(1-\frac{\eta}{1+\delta^{2}}\right)^{2}+\left(\frac{n \delta}{1+\delta^{2}}\right)^{2}\right\}^{\frac{1}{2}}-\left(1-\frac{\eta}{1+\delta^{2}}\right)\right]^{\frac{1}{2}},
\end{aligned}
$$

when there is no magnetic field.

Equations (1), (2), (4a) and (4b) give the complex reflection coefficient $R$ as a function of $\eta$ and $\delta$. They can be inverted to give $\eta$ and $\delta$ as functions of the modulus, $|R|$, and phase angle, $\theta$, of the reflection coefficient $R=|R| e^{j \theta}$.

For example, let us consider first the case for $\delta=0$. Eqs. (4a) and $(4 b)$ then reduce to

$$
\begin{aligned}
& \left.\begin{array}{l}
\alpha=k_{0} \sqrt{1-\eta}, \\
\beta=0,
\end{array}\right\} \text { for } \eta<1, \\
& \left.\begin{array}{l}
\alpha=0, \\
\beta=k_{0} \sqrt{\pi-1},
\end{array}\right\} \text { for } \eta>1 .
\end{aligned}
$$

Substitution of Eq. (5b) into Eq. (I) gives

$$
R=\frac{1+j \sqrt{\eta-1}}{1-j \sqrt{\eta-1}} .
$$

The phase angle, $\theta$, is immediately obtained from Eq. (6):

$$
\theta=2 \tan ^{-1} \sqrt{\eta-1},
$$

which indicates that $\theta$ tends to $\pi$ as $\eta$ goes to $\infty$. Thus, a plasma of infinite density is equivalent to a perfect conductor as far as the reflection coefficient is concerned. It is sometimes convenient to use 
$\varphi \equiv \pi-\theta$ rather than $\theta . \mathrm{Eq} \cdot(7)$ is then written as,

$$
\varphi=2 \tan ^{-1} \frac{1}{\sqrt{r_{1-1}}}
$$

Eq. (8), solved for $\eta$, yields,

$$
\begin{aligned}
\eta & =\frac{1}{\sin ^{2} \varphi / 2} \\
& \approx \frac{1}{\tan ^{2} \frac{\varphi}{2}} \text { for } \eta \gg I
\end{aligned}
$$

The Eq. (9) shows that the normalized density $\eta$ is readily obtained by measuring the phase angle $\varphi$. The value of $\varphi$ is conventionally calculated from the shift of the standing wave appearing in the vacuum side. The standing wave $\mathrm{E}$ is expressed as,

$$
\begin{aligned}
E & =e^{-j k_{0} z}+R e^{j k_{0} z} \\
& =2 e^{j \frac{\theta}{2}} \cos \left(k_{0} z+\frac{\theta}{2}\right) .
\end{aligned}
$$

AccordingIy, the shift of the standing wave minimum, $S$, as referred to the position for the perfect conductor, is obtained by putting $k_{0} S+\frac{\theta}{2}=\frac{\pi}{2}$. Then,

$$
k_{0} S=\frac{\varphi}{2}=\tan ^{-1} \frac{1}{\sqrt{\eta-1}}
$$

This shift of the standing wave can be expressed in terms of the attenuation or penetration distance, $d$, defined by

$$
\begin{aligned}
d & \equiv \frac{1}{\beta}, \\
& =\frac{1}{k_{0} \sqrt{\eta-1}} \text { for } \eta>I \text { and } \delta=0
\end{aligned}
$$

From Eqs. ( 8 ), (11) and (12b), we obtain the following relation:

$$
k_{0} S=\tan ^{-1}\left(k_{0} d\right)
$$


Thus the shift of the standing wave is equal to the penetration depth of the incident wave into the plasma, provided $k_{0} d \ll I$.

For plasmas with $\delta \neq 0$, things are somewhat involved as is seen in Eq. (4). However the expressions for $\eta$ and $\delta$ in terms of $|R|$ and $\delta$ have been derived for $\eta \gg I+\delta^{2}$ : they are, ${ }^{1}$

$$
\begin{gathered}
\eta=\frac{4}{\tan ^{2} \varphi-(1-|R|)^{2}}, \\
\delta^{2}=\left[\frac{1+(1-|R|)^{2} / \tan ^{2} \varphi}{1-(1-|R|)^{2} / \tan ^{2} \varphi}\right]^{2}-1 .
\end{gathered}
$$

Noting $k_{0} S=\varphi / 2$ and $\tan \frac{\varphi}{2} \cong \frac{1}{2} \tan \varphi$, one can rewrite Eqs. (14a) and (14b) in the following forms,

$$
\begin{gathered}
\eta=\frac{1}{\tan ^{2} k_{0} S-(I-|R|)^{2} / 4} \\
\delta^{2}=\left[\frac{I+(I-|R|)^{2} / 4 \tan ^{2} k_{0} S}{1-(1-|R|)^{2} / 4 \tan ^{2} k_{0} S}\right]^{2}-1 .
\end{gathered}
$$

The values of $\eta$ against $\tan \varphi$ are shown in Fig. I for various values of $\delta$. It is seen that the correction to $\eta$ due to finite $\delta$ is relatively small. For time varying plasmas, it is more convenient to utilize a suitable couple of the standing wave signal. The normalized amplitude, $h_{z}$, of the standing wave detector which is assumed to have an ideal square characteristic, is given by,

$$
h_{z}=\frac{1}{4}\left|I+R \cdot e^{-j 2 k_{0}|z|^{2}}\right|^{2}
$$

The numerical factor $1 / 4$ is introduced in order to normalize all standing wave amplitudes to unit maximum for $\mathrm{R}=1$. 
Eq. (16) can be solved for $\eta$ and $\delta$ with two $h_{z}{ }^{\prime}$ s observed at two different positions. While the choice of these points is to some extent arbitrary, the four points shown in Fig. 2 are preferred to get concise expressions for $\eta$ and $\delta$. After some calculations and suitable approximations, the following relations are deduced:

a) For $\eta \gg 1+\delta^{2}$,

$$
\begin{aligned}
\eta & =\frac{4}{\left(h_{3}-h_{4}\right)^{2}-\left\{1-\left(h_{3}+h_{4}\right)\right\}^{2}} \\
\delta^{2} & =\left[\frac{\left(h_{3}-h_{4}\right)^{2}+\left\{1-\left(h_{3}+h_{4}\right)\right\}^{2}}{\left(h_{3}-h_{4}\right)^{2}-\left\{1-\left(h_{3}+h_{4}\right)\right\}^{2}}\right]^{2}-1 .
\end{aligned}
$$

The above equations reduce to

$$
\begin{aligned}
& \eta \simeq \frac{4}{\left(h_{3}-h_{4}\right)^{2}}, \\
& \delta \simeq \frac{2}{\left(h_{3}-h_{4}\right)} \cdot\left\{1-\left(h_{3}+h_{4}\right)\right\},
\end{aligned}
$$

for $\delta^{2} \ll 1$. Eq. (18a) can be slightly modified to give,

$$
\frac{\eta^{2}}{r_{1}-1}=\frac{4}{\left(h_{3}-h_{4}\right)^{2}}
$$

which is still applicable even for $\eta>1$, provided $\delta \ll 1$.

b) For $\eta>1$ and $\delta^{2} \ll 1$,

$$
\begin{aligned}
& \eta \simeq 1+\frac{h_{2}}{h_{1}} \\
& \delta \simeq \sqrt{\frac{h_{2}}{h_{1}}} \cdot\left\{1-\left(h_{1}+h_{2}\right)\right\}
\end{aligned}
$$

In deriving Eqs. (19a) and (19b), $\alpha$ and $\beta$ have been approximated by

$$
\alpha \simeq k_{0} \eta_{\delta} / 2 \sqrt{\Upsilon_{1-1}}
$$




$$
\beta \simeq k_{0} \sqrt{\eta-1}
$$

Eqs. (18c) and (19a) simplify respectively to

$$
\frac{\eta^{2}}{\eta-1}=\frac{1}{\left(1 / 2-h_{4}\right)^{2}}=\frac{1}{\left(h_{3}-1 / 2\right)^{2}}
$$

and

$$
\eta=\frac{1}{h_{1}}
$$

when $\delta=0$. Eq. (2lb) will be seen to be exactly equivalent to Eq. (9a).

Since the phase and attenuation constants in a plasma filled waveguide through which the microwave excited to $\mathrm{TE}_{10}$ mode propagates can be obtained by merely replacing $\eta$ in Eqs. (4a) and (4b) by $\eta /\left(1-\lambda^{2} / \lambda^{2}{ }_{c}\right)$ where $\lambda$ and $\lambda_{c}$ are the wavelength in free space and the cutoff wavelength of the waveguide, the formulas in this chapter and in the following chapters can be applied for a waveguide system provided $\eta$ is replaced by $N\left(1-\lambda^{2} / \lambda^{2}{ }_{c}\right)$. Eqs. (19a) and (19b) have been successfully used to determine $\eta$ and $\delta$ in a recombination controlled afterglow plasma which was produced in an $\mathrm{X}$-band rectangular waveguide. In this experiment an electron density as high as $1013 / \mathrm{cc}$ has been measured.4

\section{Some Considerations for Reducing Experimental Errors}

\subsection{Difference Method}

In order to be able to measure a density as high as $\eta=10^{4}$ one has to measure within a reasonable error range a value of $\left(h_{3}-h_{4}\right)$ as small as $2 \times 10^{-2}$ or 1.1 degree in the phase angle. $\eta=10^{4}$ also corresponds to the shift of the standing wave minimum of $S=(\lambda / 2 \pi) \times 10^{-2} \mathrm{~cm} \simeq 5 \times 10^{-3} \mathrm{~cm}$ for an $X$-band microwave of $\lambda=3 \mathrm{~cm}$. Thus the correct location of the standing wave detectors is of the primary importance. 
However, the errors in $\eta$ and $\delta$ due to the slight mis-location of the detectors can be to some extent avoided by a sort of difference method when 1t, is possible to get an electron density much higher than those which are to be measured. Such a condition as above is often fulfilled in shock experiments.

Let $h_{3}{ }^{\prime}$ and $h_{4}{ }^{\prime}$ be the standing wave amplitudes for a plasma whose density is much higher than those of interest; then $\eta$ and $\delta$ can be expressed in terms of

$$
\begin{aligned}
& \Delta h_{3} \equiv h_{3}-h_{3}^{\prime}, \\
& \Delta h_{4} \equiv h_{4}-h_{4}^{\prime},
\end{aligned}
$$

as follows;

$$
\begin{aligned}
\eta & =\frac{4}{\left(\Delta h_{3}-\Delta h_{4}\right)^{2}-\left(\Delta h_{3}+\Delta h_{4}\right)^{2}}, \\
\delta^{2} & =\left\{\frac{\left(\Delta h_{3}-\Delta h_{4}\right)^{2}+\left(\Delta h_{3}+\Delta h_{4}\right)^{2}}{\left(\Delta h_{3}-\Delta h_{4}\right)^{2}-\left(\Delta h_{3}+\Delta h_{4}\right)^{2}}\right\}^{2}-1,
\end{aligned}
$$

provided $\delta / \sqrt{\eta} \gg \delta^{1} / \sqrt{\eta^{1}}$ and $(2 \pi / \lambda)\left(\Delta x_{3}+\Delta x_{4}\right) \ll 1$, where $\Delta x_{3}$ and $\Delta x_{4}$ are the errors in the positions of the standing wave detectors for $h_{3}$ and $h_{4}$ respectively, and $\delta^{\prime}$ and $\eta^{\prime}$ are the values for the reference high density plasma.

Eqs. (23a) and (23b) reduce to

$$
\begin{aligned}
& \eta \approx \frac{4}{\left(\Delta h_{3}-\Delta h_{4}\right)^{2}}, \\
& \delta \approx 2 \cdot \frac{\Delta h_{3}+\Delta h_{4}}{\Delta h_{3}-\Delta h_{4}}
\end{aligned}
$$


for $\delta^{2} \ll 1$. Note that $\left(\Delta h_{3}+\Delta h_{4}\right)<\left(\Delta h_{3}-\Delta h_{4}\right)$ because of $\Delta h_{3}>0$ and $\Delta \mathrm{h}_{4}<0$.

Eq. (24a) has been used to evaluate the electron densities from $10^{13}$ to $3 \times 10^{15} / \mathrm{cc}$ in a plasma produced by an electromagnetically driven shock wave into argon. 5

\subsection{Effect of Power Losses Along the Path Between the Plasma Boundary}

\section{and the Standing Wave Detectors}

The power losses, such as the circuit loss due to the finite conductivity of the waveguide or some scattering loss between a horn antenna and the plasma boundary, have to be taken into account to get the correct values of $\eta$ and $\delta$. When these losses are of pure dissipative type and accordingly give rise to no phase shift, the standing wave signal, $h_{z}$, given by $\mathrm{Eq} \cdot(16)$ is modified as follows:

$$
h_{z}=\frac{1}{4}\left|1+\rho R e^{-j 2 k_{0}|z|^{2}}\right|^{2}
$$

Here, a correction factor, $p$, which is a real number less than one, is introduced to account for these power losses. The similar calculations as in the preceding chapter result in,

$$
\begin{gathered}
\eta \approx \rho^{2} \frac{4}{\left(\hat{h}_{3}-h_{4}\right)^{2}}, \\
\delta \approx \frac{\rho}{h_{3}-h_{4}} \cdot\left[1+\frac{1}{\rho^{2}}\left\{1-2\left(h_{3}+h_{4}\right)\right\}\right],
\end{gathered}
$$

provided $\eta>1$ and $\delta^{2}<1$.

Alternate expressions for $\eta$ and $\delta$ in terms of $h_{1}$ and $h_{2}$ are possible: they are, 


$$
\begin{aligned}
& \eta=I+h_{20} / h_{10}, \\
& \delta=\frac{h_{20}}{h_{10}} \cdot\left\{I-\left(h_{10}+h_{20}\right)\right\},
\end{aligned}
$$

with

$$
\begin{aligned}
& h_{10}=\frac{1}{2 p}\left\{\sqrt{2\left(h_{1}+h_{2}\right)-1}-\left(h_{2}-h_{1}\right)\right\}, \\
& h_{20}=\frac{1}{2 p}\left\{\sqrt{2\left(h_{1}+h_{2}\right)-1}+\left(h_{2}-h_{1}\right)\right\} .
\end{aligned}
$$

When $\delta=0, \mathrm{Eq} \cdot(27 a)$ reduces to

$$
\eta=\frac{p}{h_{1}-\frac{1}{4}(I-p)^{2}}
$$

by noting that $h_{1}+h_{2}=\frac{1}{2}\left(1+\rho^{2}\right)$ for $\delta=0$.

Eqs. (26a) and (26b) can again be rewritten in terms of $\Delta h_{3}$ and $\Delta h_{4}$ defined in $\mathrm{Eq} \cdot(22)$;

$$
\begin{aligned}
& \eta=\rho^{2} \cdot \frac{4}{\left(\Delta h_{3}-\Delta h_{4}\right)^{2}} \\
& \delta=\frac{2}{\rho} \cdot \frac{\Delta h_{3}+\Delta h_{4}}{\Delta h_{3}-\Delta h_{4}}
\end{aligned}
$$

Eqs. (26), (29) and (30) reduce to Eqs. (18), $21 \mathrm{~b}$ and (24) respectively when $p=1$.

Note that the introduction of the correction factor $\rho$ does not result in any change in the shift of the standing wave minimum $S$, as is clear from Eq. (25) and the definition of $p$.

4. Further Extension of Measurable Density Range

As is well known in transmission theory, a small phase shift can be expanded by making use of a suitable buffer dielectric plate of $\lambda_{\epsilon} / 4$ 
thickness. This idea is here utilized to extend further the measurable density range. The reflection coefficient $R$ at the surface of the dielectric plate, whose other side is in contact with plasma as is shown in Fig. 3, can be easily calculated to give,

$$
R=\frac{k_{3} / k_{0}-\left(k_{2} / k_{0}\right)^{2}}{k_{3} / k_{0}+\left(k_{2} / k_{0}\right)^{2}}
$$

where

$$
\begin{aligned}
& \mathrm{k}_{3}=-j \mathrm{k}_{0} \sqrt{\tau_{1-1}} \\
& \mathrm{k}_{2}=\mathrm{k}_{0} \sqrt{\varepsilon}
\end{aligned}
$$

and $\varepsilon$ is the specific dielectric constant of the plate.

Substitution of Eq. (32) into (31) gives

$$
R_{1}=-\frac{1+j \sqrt{\eta-1} / \varepsilon}{1-j \sqrt{\eta-1} / \varepsilon},
$$

which, when re-evaluated at a point off the dielectric boundary by a distance $\lambda / 4$, turns out to be

$$
R_{1}=\frac{1+j \sqrt{\eta-1} / \varepsilon}{1-j \sqrt{\eta-1} / \varepsilon}
$$

The shift of the standing wave minimum, $S_{1}$, is now given by

$$
\begin{aligned}
k_{0} S_{1} & =\tan ^{-1} \cdot \frac{\varepsilon}{\sqrt{\eta-1}} \\
& \approx \frac{\varepsilon}{\sqrt{\eta}} \text { for } \eta \gg 1
\end{aligned}
$$

$\mathrm{Eq}$. (35) shows that $\mathrm{S}_{1}$ is nearly $\varepsilon$ times $\mathrm{S}$ of $\mathrm{Eq}$. (11). Accordingly the maximum, measurable value of $\eta$ is risen up by a factor $\varepsilon^{2}$. Eq. (35) can be easily generalized to the case where $n$ dielectric plates are placed in front of the plasma boundary, each plate being separated by a distance 
$\lambda / 4$ from its neighbors. Then the shift of the standing wave minimum, $\mathrm{S}_{\mathrm{n}}$, observed in front of the $\mathrm{n}$-th plate is given by

$$
k_{0} S_{n}=\tan ^{-1} \frac{\varepsilon^{n}}{\sqrt{1-1}} \text {. }
$$

It is noted that the first dielectric plate does not necessarily have to be in contact with the plasma; the plate located at distance $\lambda / 2$ from the plasma boundary gives the same result.

The technique described here can be also applied to plasmas whose densities are less than critical. When the plasma shown in Fig. 3 is replaced by a plasma layer of thickness $L$, of density $\eta<1$ and whose other boundary is made in contact with a perfect conductor, the relection coefficient evaluated at a distance $\lambda / 4$ off the dielectric plate is given by

$$
R_{1}^{\prime}=\frac{1+j \sqrt{1-T} / \varepsilon \cdot \tan k_{0} I \sqrt{1-\eta}}{1-j \sqrt{1-T} / \varepsilon \cdot \tan k_{0} I \sqrt{1-\eta}} .
$$

If we choose $k_{0} L=2 m \pi$ where $m$ is an interger, we have tan $k_{0} L \sqrt{1-\eta} \cong m m \eta$ for $\eta \ll 1$. Thus the shift of the standing wave minimum, $S_{1}^{\prime}$, is given by

$$
\mathrm{k}_{\mathrm{O}} \mathrm{S}_{I}^{\prime} \approx \mathrm{m} \pi \varepsilon \eta,
$$

which is $\varepsilon$ times larger than that without the dielectric plate. The minimum, measurable value of $\eta_{\text {is }}$ therefore lowered by a factor $\epsilon$. With $n$ plates, being separated by a distance $\lambda / 4$ from each other, the shift of minimum observed in front of the n-th plate is,

$$
k_{0} S_{n}^{\prime}=m \pi \varepsilon^{n} \eta
$$

The other boundary of the plasma layer needs not necessarily be in contact with the metallic conductor; the standing wave can be constructed from two signals in a bridge type circuit. One signal is taken directly 
from the microwave oscillator, while the other is taken through the plasma layer. Thus the present technique covers a wide range of measurable density, for instance, from $\eta=10^{-4}$ to $10^{6}$ when two dielectric plates of $\epsilon=3.7$ are used for a plasma layer whose thickness is $\lambda / 2$. In a waveguide, $\epsilon$ has also to be replaced by $\left(\epsilon-\lambda^{2} / \lambda_{c}{ }^{2}\right) /\left(1-\lambda^{2} / \lambda_{c}{ }^{2}\right)$.

\section{Microwave Reflection Probe}

Hitherto we have been mainly concerned about plane wave analyses. However additional problems arise when a microwave probe, as is illustratéd in Fig. 4, is used to obtain the local electron density of a plasma in free space.

First of all, a part of incident microwave power is reflected at the probe end even when there is no plasma outside the probe, because of the abrupt change of geometry. Of course this type of reflection can be eliminated by using a suitable matching device such as three stubs or E-H tuner. The problem is whether the tuner, once matched for free space, has to be re-adjusted when the probe is inserted into plasmas. An analysis has been made of the equivalent circuit based on the theory of the transmission lines; the result shows that the overall reflection coefficient is given by a product of a constant phase factor and the reflection coefficient of the plasma boundary, provided the system is matched initially for free space. This constant phase factor can experimentally be corrected for by referring phase to the metallic short case after the matching for free space is achieved.

The second and more serious problem is how to handle the distortion of the electric field outside the probe. Galejs has calculated the 
admittance of a waveguide radiating into plasma, ${ }^{7}$ and Wait has given a formal solution to the radiation field outside a slotted conducting plane in a plasma environment. ${ }^{8}$ In both cases, the electromagnetic wave propagates into the plasma through a slot antenna which is located in a perfectly conducting infinite plane.

Instead of having recourse to the rigorous, but cumbersome mathematics, we develop here an heuristic and semi-empirical method. ${ }^{9}$ We first assume that the fringing field in vacuum can be expressed approximately by the field produced by a line dipole placed at distance $Z_{0}$ behind the probe end. Then the field strength at distance $z$ from the probe end is given by $E_{0} Z_{0}^{2} /\left(z+Z_{0}\right)^{2}$. This has to be multiplied by the attenuation factor $\exp (-z / d)$ in order to give the field when plasma is present. Thus, to a first approximation, we obtain

$$
E(z)=E_{0}\left(\frac{Z_{0}}{z+Z_{0}}\right)^{2} \cdot \exp \left(-\frac{z}{d}\right)
$$

for the fringing field in plasma, where $E_{O}$ is the value at $z=0$, and $Z_{0}$ depends presumably on the ratio of the wavelength to the characteristic probe dimension. On the other hand, we can also express $E(z)$ formally in terms of an effective penetration depth, d', as follows:

$$
E(z)=E_{0} \exp \left(-\frac{z}{d^{\prime}}\right)
$$

To define $d^{\prime}$ in terms of $d$ and $z_{0}$, the two expressions for $E(z)$ are set equal at some point $z=Z_{1}$. While the choice of such a point seems arbitrary, we may argue on physical grounds that the best choice of $Z_{1}$ would be $z_{1}=d$, where the field is roughly $e^{-1}$ times $E_{0}$. The result is: 


$$
d^{\prime}=d\left[1+2 \ln \left(1+d / z_{0}\right)\right]^{-1} \text {. }
$$

This equation reduces to the correct limit, $d^{\prime} / d=1$, for large $\eta$ or small d, for it is just when $d<z_{0}$ that fringing field effects are expected to be negligible. For d large, d'/d $<1$ expresses the fact that a smaller effective penetration depth is here used to compensate for the field decrease caused by the effect of geometry. With Eq. (42), one can easily obtain the reflection coefficient, noting $d^{\prime}=1 / \beta^{\prime}$ and $\mathrm{d}=1 / \beta$. Or one may make use of $\mathrm{Eq} \cdot(12 \mathrm{~b})$ to compare $\eta^{\prime}$ with $\eta_{\text {, where }}$ II is the actual density just outside the probe and $\eta^{\prime}$ includes the fringing field effect. In Fig. 5 are shown the values of $d / d$ and $\eta_{1} / \eta$ against $d / z_{0}$ for various values of $z_{0} / \lambda$. The value of $z_{\circ}$ remains to be determined experimentally. And this was done with a uniform argon afterglow plasma produced in a cylindrical waveguide. The phase shift was simultaneously measured with an X-band microwave at the two ends of the chamber. At one end, the chamber was attached to a cylindrical waveguide of the same diameter as the discharge tube so that fringing fields are absent. The microwave probe was used at the other end. It consisted of a standard $R G-52 / \mathrm{U}$ waveguide tapered to a $3 \mathrm{~mm}$ height so that the plasma in the cylindrical chamber is considered as a plasma in free space for the probe. The calculated and observed values of $\eta^{\prime}$ showed good agreement within the experimental range of $\eta_{i}<10$ when $z_{0} / \lambda_{g}$ was chosen equal to 0.63 .

A $\mathrm{K}$-band microwave probe of the cross section $7 \mathrm{x} I \mathrm{~mm}^{2}$ was successfully used to determine the density profile of an argon arc plasma. The 
densities measured by the probe fitted quite well with those obtained by other techniques such as the Stark broadening of $\mathrm{H} \beta$ line and Langmuir probe. ${ }^{6}$

\section{Reflection by Inhomogeneous Plasma}

Need sometimes arises to take account of inhomogeneity of the electron density along the direction normal to the boundary surface, when one wants, for instance, to measure the density profile of a cylindrical plasma whose characteristic diameter is of a comparable order of magnitude as the wavelength of the probing signal.

To choose a simple case for illustration, let us consider a linear density ramp with a sharp cut at $\mathrm{z}=0$ where the reflection probe is located, as is depicted in Fig. 6. Since the gradient of the electron density is parallel to the direction of the propagation of the incident wave, the wave equation for the electric field strength, E, is simply given by $^{10}$

$$
\frac{\mathrm{d}^{2} \mathrm{E}}{\mathrm{dz^{2 }}}+k_{0}^{2}(1-\eta) \mathrm{E}=0
$$

with

$$
\begin{array}{ll}
\eta=0 & \text { for } z<0, \\
\eta=\eta_{0}+\frac{z}{z_{0}} & \text { for } z \geq 0 .
\end{array}
$$

The solutions for the region $I$ and II, in Fig. 6, is given by

$$
\begin{gathered}
E_{I}=e^{-j k_{0} z}+R e^{j k_{0} z}, \\
E_{I I}=A \sqrt{x} I_{1 / 3}(\xi)+B \sqrt{x} I_{-I / 3}(\xi),
\end{gathered}
$$


where

$$
\begin{aligned}
& x \equiv \eta-1, \\
& \xi \equiv \frac{2}{3} k_{0} z_{0} x^{3 / 2},
\end{aligned}
$$

and $I_{ \pm I / 3}(\xi)$ are the modified Bessel functions of orders $\pm 1 / 3$. The boundary condition that $E_{I I}$ has to vanish at $z \rightarrow \infty$ requires $A=-B$, since the asymptotic form of $I_{ \pm 1 / 3}(\xi)$ is given by ${ }^{11}$

$$
I_{ \pm I / 3}(\xi) \underset{\xi \rightarrow \infty}{\longrightarrow}(2 \pi \xi)^{-I / 2}\left[e^{\xi}+e^{-\xi} e^{-j\left(\frac{1}{2} \pm \frac{1}{3}\right) \pi}\right] \text {. }
$$

The reflection coefficient $R$ is obtained from Eqs. (45) and (46) by putting $E_{I}=E_{I I}$ and $\frac{d E_{I}}{d z}=\frac{d E_{I I}}{d z}$ at $z=0$.

After some calculations, we obtain

$$
R=\frac{1-j K}{1+j K}
$$

where

$$
K=\left[\frac{x I_{-2 / 3}(\xi)+x I_{2 / 3}(\xi)}{\sqrt{x} I_{I / 3}(\xi)-\sqrt{x} I_{-I / 3}(\xi)}\right]_{z}=0 .
$$

When $k_{0} Z_{0}\left(\eta_{0}-1\right)^{3 / 2} \gg I$, in other words, when the density gradient is very small and/or $\eta_{0}$ is very high, Eq. (49b) reduces to

$$
K \approx-\sqrt{\eta_{0}-1}\left\{1+\frac{1}{4 k_{0} Z_{0}} \cdot \frac{1}{\left(\eta_{0}-1\right)^{3 / 2}}\right\} .
$$

Hence, we have

$$
R \cong \frac{1+j \sqrt{\eta_{0}-1}\left\{1+\frac{1}{4 k_{0} z_{0}} \cdot \frac{1}{\left(\eta_{0}-1\right)^{3 / 2}}\right\}}{1-j \sqrt{\eta_{0}-1}\left\{1+\frac{1}{4 k_{0} z_{0}} \cdot \frac{1}{\left(\eta_{0}-1\right)^{3 / 2}}\right\}} .
$$


As is expected, Eq. (5I) reduces to $E q$. (6) when $Z_{0} \rightarrow \infty$, that 1 , when the density gradient approaches zero.

It can be shown that a WKB-type approximation method leads to the same result as Eq. (5I). When the change in the electron density over a range considered is very small compared to the value at the boundary, the electric field can be approximated by

$$
\mathrm{E}_{I I} \approx \mathrm{C} e^{-\mathrm{k}_{0} \int_{0}^{\mathrm{z}} \sqrt{\eta-I} \mathrm{dz}},
$$

where $\mathrm{C}$ is a constant. The effective attenuation distance, $\mathrm{d}$, is then defined by

$$
k_{0} \int_{0}^{d} \sqrt{1-1} \cdot d z=1
$$

The integrand can be expanded as follows,

$$
\begin{aligned}
\sqrt{\eta-1} & =\sqrt{\eta_{0}+\frac{z}{z_{0}}-1} \\
& \approx \sqrt{\pi_{0}-1}\left\{1+\frac{z}{2 z_{0}\left(\eta_{0}-1\right)}\right\} .
\end{aligned}
$$

Inserting Eq. (54) into Eq. (53) and solving for d, we obtain,

$$
\frac{1}{d} \approx k_{0} \sqrt{\eta_{0}-1}\left\{1+\frac{1}{4 k_{0} z_{0}} \cdot \frac{1}{\left(\eta_{0}-1\right)^{3 / 2}}\right\},
$$

provided $4 k_{0} Z_{0}\left(\eta_{0}-I\right)^{3 / 2}>I$. Since $\alpha=I / \beta$ and $R=\left(k_{0}+j \beta\right) /\left(k_{0}-j \beta\right)$ for a loss-less plasma, Eq. (55) gives the same reflection coefficient as $\mathrm{Eq} \cdot(5 \mathrm{I})$. 
7. Reflection by Boundary Layer with Density Gradient

In the preceding chapters, it has been assumed that the reflection always takes place at a well defined boundary surface across which the electron density changes stepwise. However, there exists always a finite boundary layer in an actual plasma, at least, of the order of the Debye length. The boundary layer may become still thicker due to diffusion into the waveguide, when a waveguide probe is inserted into a plasma and when it is impossible, due to heat damage or charge accumulation which might cause some undesirable effect on the plasma, to use a thin insulator to divide the plasma and vacuum.

Microwave reflection by a non-uniform boundary has been treated by many authors: Taylor has calculated reflections at linear density ramps $^{12}$ and also at an inverse parabolic density profile, ${ }^{13}$ Wait has given a formal solution for a stratified plasma, ${ }^{14}$ Albini and Jahn have made numerical calculations of reflection and transmission coefficients for linear and "kinked" ramps, connecting uniform plasmas and for trapezoid geometries, ${ }^{\circ}$ and recently an exponential electron distribution has been attacked by Yen.15

Our problem here is to seek the phase shift of the reflection coefficient caused by the existence of a relatively thin boundary layer. Jahn has already given an answer for a special case. He has derived the following formula for the geometry shown in Fig. $7,{ }^{16}$ 


$$
R_{J}=\frac{\Omega_{2}^{*}+\frac{k_{0}}{2 k_{2}^{*}}\left[\left(1-\Omega_{2}^{*}\right)^{2}-1\right]+\frac{j \Omega_{2}}{k_{0} z_{1}}\left(1-\frac{k_{0}}{k_{2}^{*}}\right)+\frac{j k_{0} z_{1}}{2 \Omega_{2}^{*}}\left[1-\Omega_{2}^{*}-\frac{k_{0}}{k_{2}^{*}}\left(1-\Omega_{2}^{*}\right)^{2}\right]}{\Omega_{2}^{*}+\frac{k_{0}}{2 k_{2}^{* *}}\left[1-\left(1-\Omega_{2}^{*}\right)^{2}\right]-\frac{j \Omega_{2}}{k_{0} z_{1}}\left(1+\frac{k_{0}}{k_{2}^{* k}}\right)-\frac{j k_{0} z_{1}}{2 \Omega_{2}^{*}}\left[1-\Omega_{2}^{*}+\frac{k_{0}}{k_{2}^{* *}}\left(1-\Omega_{2}^{*}\right)^{2}\right]},
$$

where

$$
\begin{aligned}
& \Omega_{2}^{*}=\frac{\omega_{p}^{2} / \omega^{2}}{1+\nu^{2} / \omega^{2}} \cdot\left(1+j \frac{\nu}{\omega}\right) \\
& k_{2}^{*}=\alpha-j \beta .
\end{aligned}
$$

In deriving $E_{q} \cdot(56)$, the Bessel functions of the forms $J_{ \pm 1 / 3}(\xi)$ have been approximated by the first few terms of their series expansions.

Jahn has also shown that, for a special case of $\eta=1$ and $\delta=0$, $R_{J}$ turns out to be

$$
R_{J}=\frac{1-j \cdot \frac{1}{2} k_{0} z_{1}}{1+j \cdot \frac{1}{2} k_{0} z_{1}},
$$

provided $k_{0} z_{1} \ll 1$. We see from $E q$. (58) that the boundary layer with linear ramp over $z_{1}$ is equivalent to a step boundary located at $z=\frac{z_{1}}{2}$ as far as the reflection is concerned.

Although Eq. (56) was obtained for $\eta_{1}<1$, it can be shown that Eq. (56) is also valid for $\eta>1$. Accordingly the statement made under Eq. (58) can be generalized for $\eta>1$. To show this, we replace $\Omega_{2}^{*}$ and $k_{2}^{*}$ in Eq. (56) by $\eta$ and $-j k_{0} \sqrt{\eta-1}$ respectively, assuming $\delta=0$, then

$$
R_{J}=\frac{A+j B}{A-j B}
$$

with

$$
A=\eta+\eta \sqrt{\eta-1} \cdot k_{o} z_{1}+\frac{(1-\eta)^{2}}{2 \eta} \cdot\left(k_{o} z_{1}\right)^{2}
$$




$$
B=\eta \sqrt{\eta-1}+\frac{1}{2}(\eta-2) k_{0} z_{1}+\frac{1-\eta}{2 \eta} \cdot \sqrt{\eta-1} \cdot\left(k_{0} z_{1}\right)^{2}
$$

The third term in Eqs. (60a) and (60b) can be neglected when $k_{o} z_{1}<1$. Hence we have

$$
R_{J}=\frac{1+\sqrt{\eta-1} \cdot k_{0} z_{1}+j\left\{\sqrt{\eta-1}+\left(\frac{\eta}{2}-1\right) k_{0} z_{1}\right\}}{1+\sqrt{\eta-1} \cdot k_{0} z_{1}-j\left\{\sqrt{\eta-1}+\left(\frac{\eta}{2}-1\right) k_{0} z_{1}\right\}}
$$

The Eq. (6I) divided by Eq. (6) yields,

$$
\frac{R_{J}}{R}=\frac{1+\frac{k_{O} z_{1}}{2} \sqrt{\eta-1}-j \frac{k_{O} z_{1}}{z}}{1+\frac{k_{0 z_{1}}}{2} \cdot \sqrt{\eta-1}+j \frac{k_{0} z_{1}}{2}}
$$

which reduces to

when

$$
R_{J} / R \cong e^{-j k_{0} z_{1}}
$$

$$
k_{0} z_{1} \sqrt{\eta-1} \ll 1
$$

Eq. (63) implies that a boundary layer with linear ramp over $z_{1}$ connecting homogeneous plasma of density $\eta$ is equivalent to a step boundary located at $z=z_{1} / 2$ of a uniform plasma having the same density.

\section{Concluding Remarks}

One of the advantages of the reflection method lies in the fact that the ratio of the sheath thickness, which is supposed to be of the order of the Debye length $\lambda_{D}$, to the penetration distance d does not depend on the electron density, but only a function of the electron temperature $T_{e}:$ since $\lambda_{\mathrm{D}} \cong \mathrm{v}_{\mathrm{T}} / \omega_{\mathrm{p}}$ and $\mathrm{d} \cong \mathrm{c} / \omega_{\mathrm{p}}$, we have $\lambda_{\mathrm{D}} / \mathrm{d} \cong \mathrm{v}_{\mathrm{T}} / \mathrm{c}$, where $c$ and $v_{T}$ are the light velocity and the mean thermal velocity of 
electrons. Thus, the thickness of the boundary layer can always be neglected compared to the penetration distance provided $V_{\mathrm{T}} / \mathrm{c} \ll I$. Although we have mainly dealt with plasmas in the absence of a magnetic field, it is clear that the formulas can be used as they stand for magnetized plasmas if geometry is chosen such that $\underset{\sim}{\mathrm{k}} \perp \underset{\sim}{\mathrm{B}}$ and $\underset{\sim}{\mathrm{E}} \| \stackrel{\mathrm{B}}{\sim}$, where $\underset{\sim}{\mathrm{B}}$ is the magnetic field.

When the extraordinary mode of propagation is utilized, that is, when $\underset{\sim}{\mathrm{k}} \perp \underset{\sim}{\mathrm{B}}$ and $\underset{\sim}{\mathrm{E}} \perp \underset{\sim}{\mathrm{B}}$, the condition $\omega_{\mathrm{c}}^{2} \ll \omega_{\mathrm{p}}^{2}$ has to be satisfied. The argument described in Chapter 6 suggests that a waveguide probe without insulating film at its end could be used for a diffusion experiment: the diffusion coefficient could be calculated from the diffusion length at the probe end.

\section{Acknowledgment}

The authors wish to express their deep appreciation to Dr. C. K. McLane of the National Bureau of Standards (NBS), Washington, D. C., for his hospitality extended to them. The discussion with him on the microwave probe was also helpful. 


\section{References}

1. S. Takeda and M. Roux, J. Phys. Soc. Japan 16, 95 (1961).

2. A. I. Anisimov, N. I. Vinogradov, V. E. Golant, and B. I. Konstantinov, Zh. Tekhn. Fiz. 30, 1010 (1960).

3. S. C. Lin et al., Phys. Fluids 5, 1633 (1962).

4. T. Tsukishima and S. Takeda, J. Appl. Phys. 33, 3290 (1962).

5. S. Takeda and T. Tsukishima, J. Phys. Soc. Japan 18, 426 (1963).

6. C. K. McLane, S. Takeda, W. E. Thomas, Jr., and R. C. Thompson, J. Appl. Phys. 35, No. 1 (Jan. 1965).

7. J. Galejs, Res. Rpt. No. 347R, Project No. 125 (1964) Jan., Applied Res. Lab., Sylvania Electronic System; also Res. Rpt. No. 346, (JuIy 1963).

8. J. R. Wait, NBS Tech. Note No. 223 (1964).

9. S. Takeda and T. Tsukishima, J. Appl. Phys. 35, 2548 (1964).

10. F. A. Albini and R. G. Jahn, J. Appl. Phys. 32, 75 (1961).

11. I. I. Schiff, Quantum Mechanics (McGraw-Hill, 1955, New York) p. 189.

12. L. S. Taylor, J. Appl. Phys. 32, 1796 (1961).

13. I. S. Taylor, IRE Trans. on Antennas and Propagation AP-9, No. 6, $582(1961)$.

14. J. R. Wait, J. Research, NBS, 65B, No. 2, April-June 1961.

15. K. T. Yen, J. Appl. Phys. 35, 290 (1964).

16. R. G. Jahn, Tech. Rpt. No. 9 (1959), Contract No. AFl8(603)-2, California Institute of Technology. p. 42. 


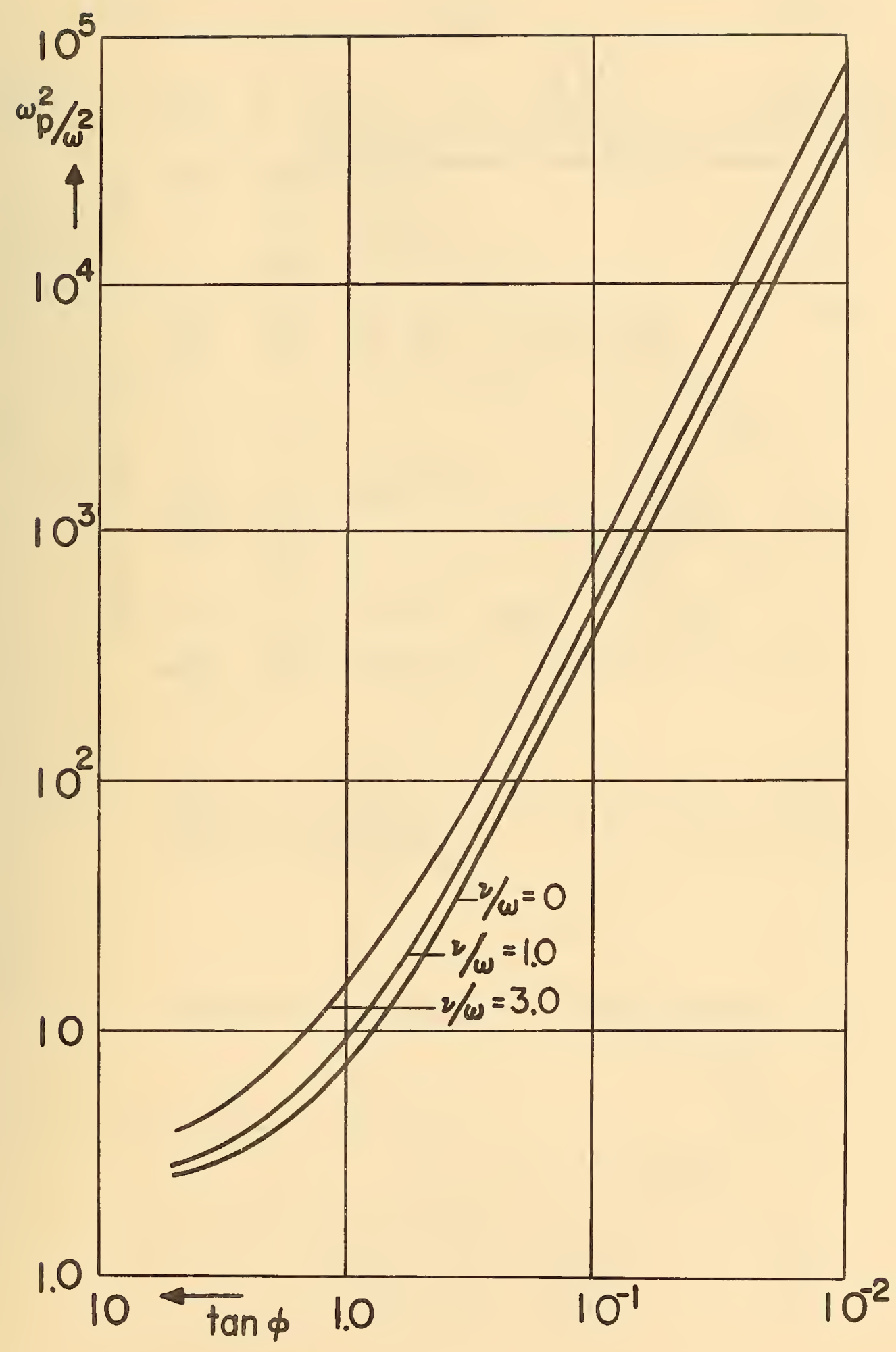

Fig. 1. Relation between $\eta_{1}$ and $\tan \varphi$ for various values of $\delta$. 


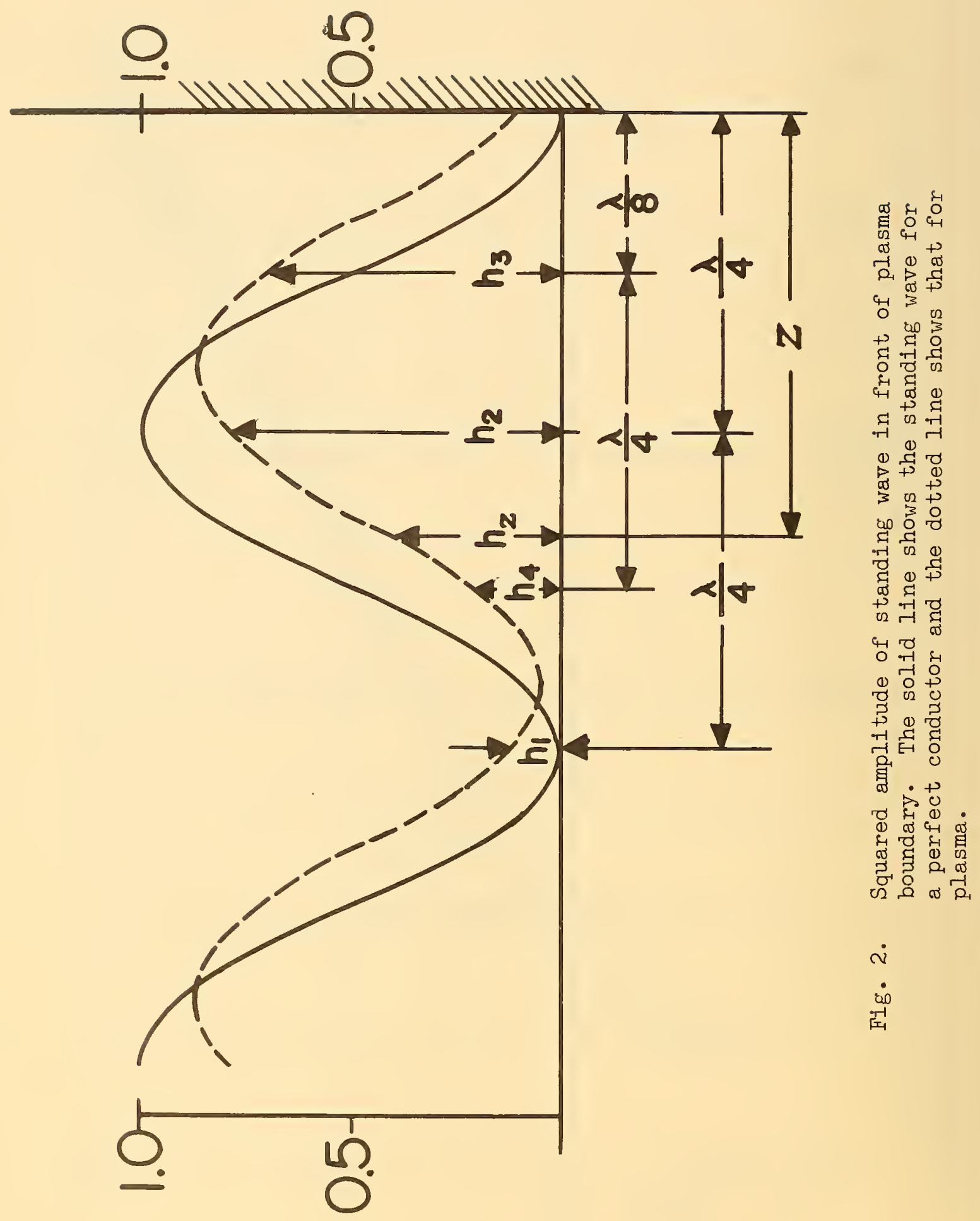



(I)
(II)
(III)

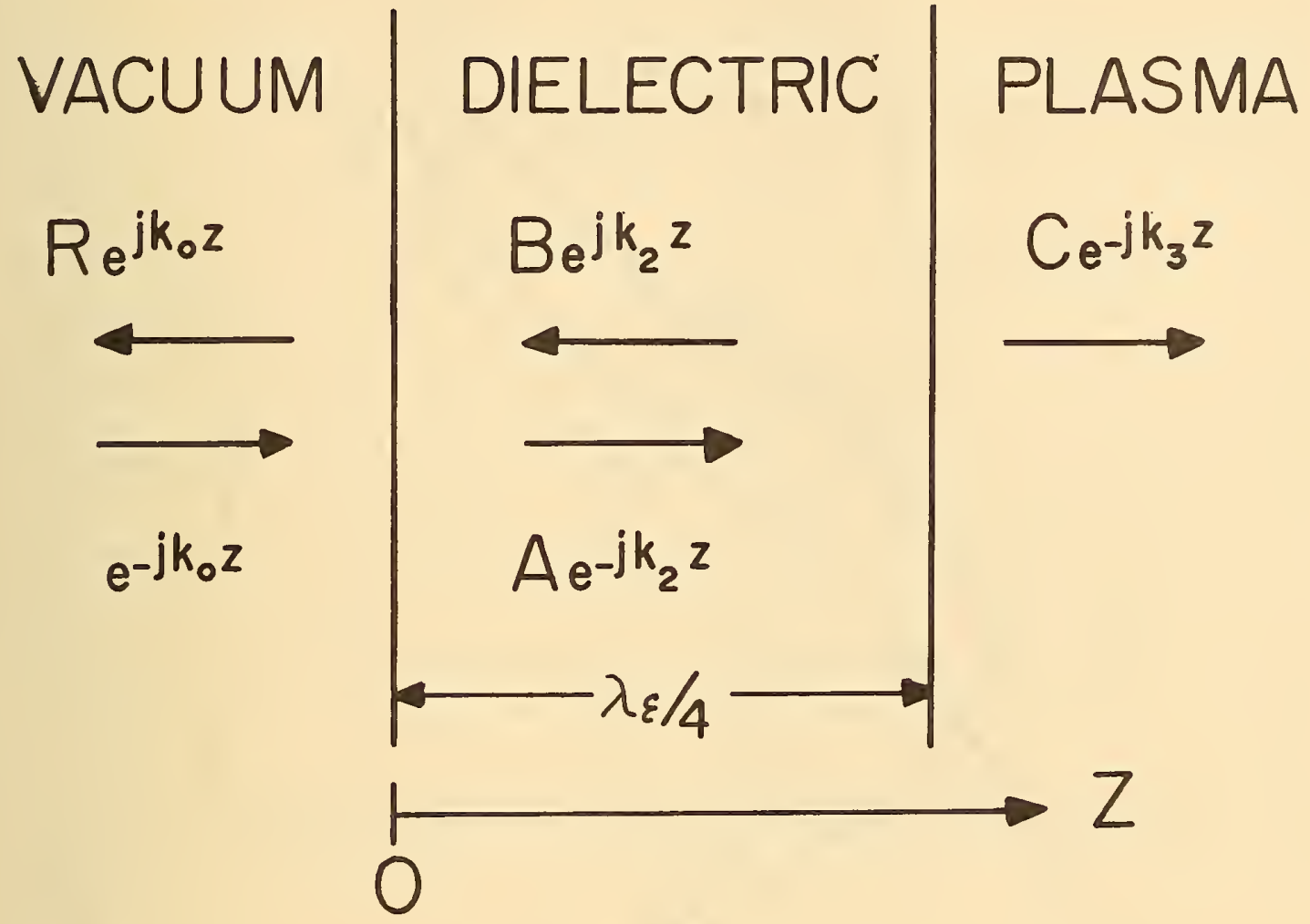

FIg. 3. Reflection by plasma through a dielectric plate whose thickness is $\lambda_{E} / 4$, where $\lambda_{E}$ is the wavelength in the
dielectric.

MICRO WAVE PROBE

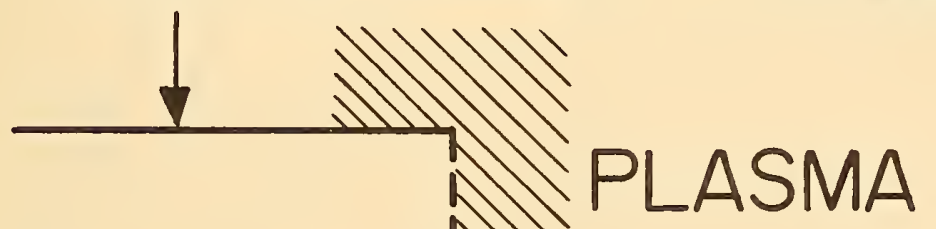

MI

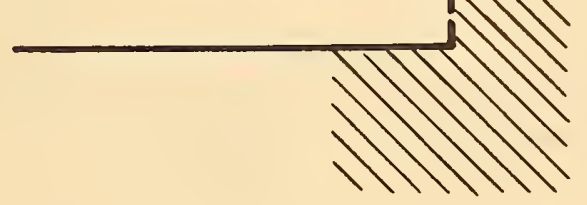

Fig. 4. Sketch of microwape probe. 


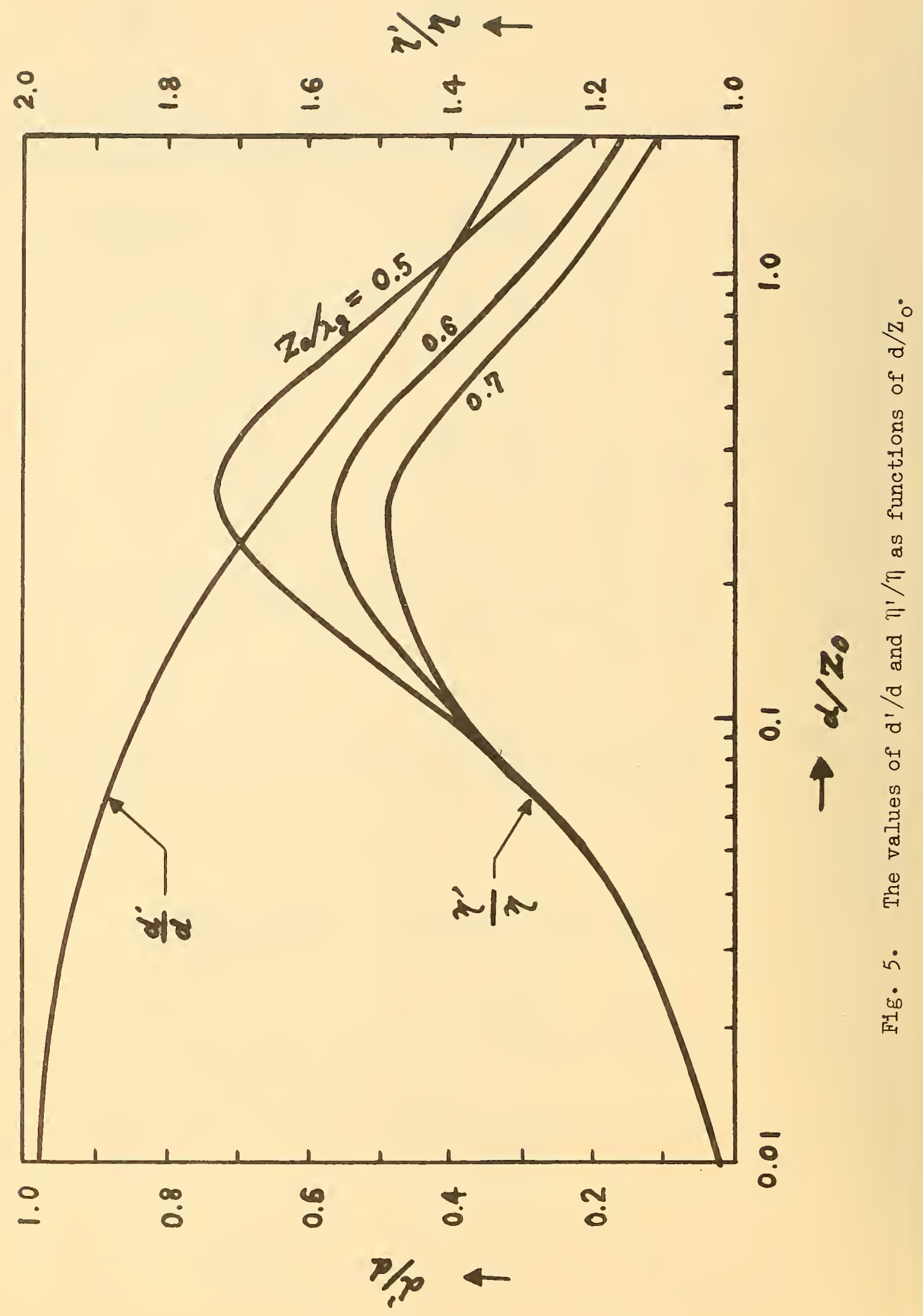




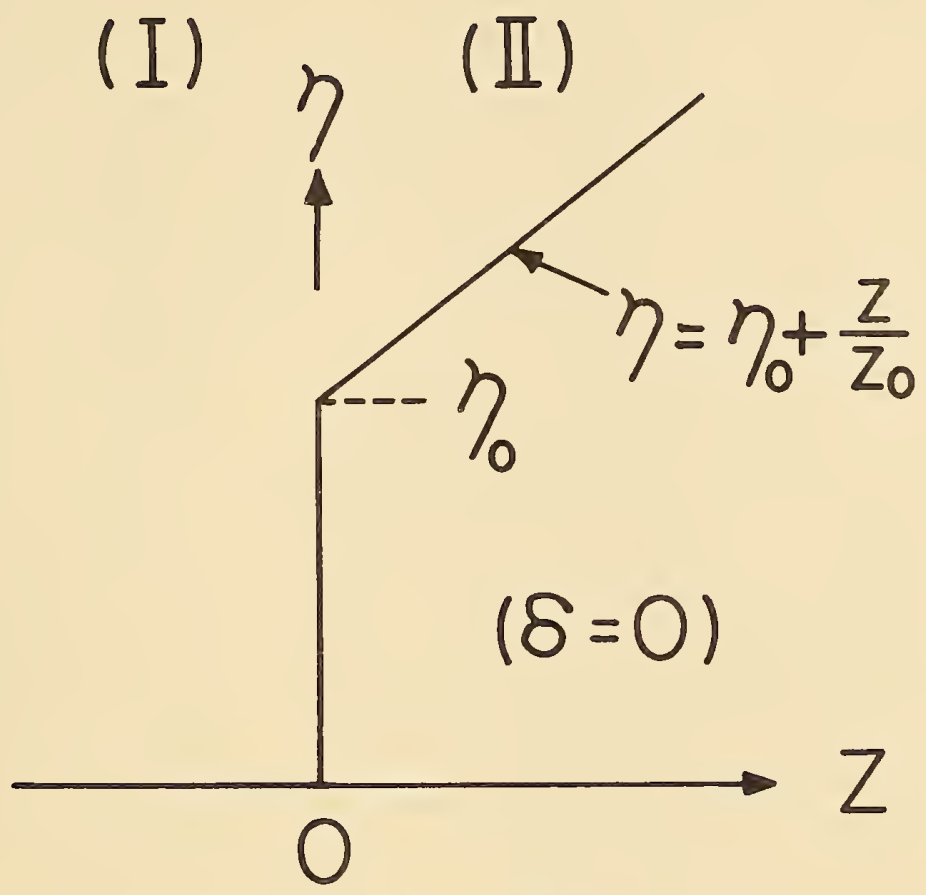

Fig. 6. Density profile of the inhomogeneous plasma having a linear ramp.

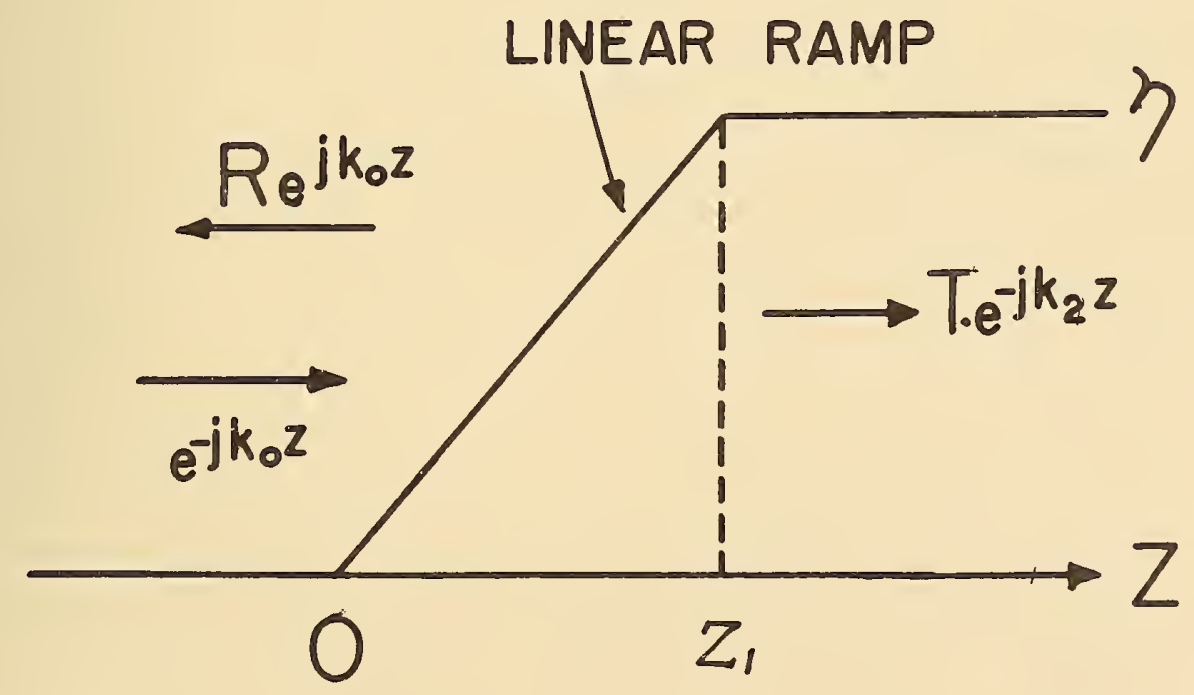

Fig. 7. Density profile of the boundary layer with a constant density gradient. 


U.S. DEPARTMENT OF COMMERCE

WASHINGTON. D.C. 20230

OFFICIAL BUSINESS
POSTAGE AND FEES PAID

U.S. DEPARTMENT OF COMMERO

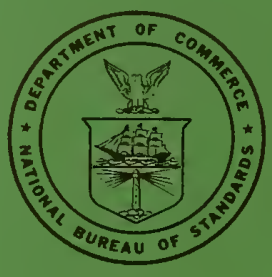

